\title{
A Meta-Analytic Review of the Efficacy of Physical Exercise Interventions on Cognition in Individuals with Autism Spectrum Disorder and ADHD
}

\author{
Beron W. Z. $\operatorname{Tan}^{1}\left(\mathbb{D} \cdot\right.$ Julie A. Pooley ${ }^{1} \cdot$ Craig P. Speelman $^{1}$
}

Published online: 13 July 2016

(c) The Author(s) 2016. This article is published with open access at Springerlink.com

\begin{abstract}
This review evaluates the efficacy of using physical exercise interventions on improving cognitive functions in individuals with autism spectrum disorder (ASD) and/or attention deficit hyperactivity disorder (ADHD). This review includes a meta-analysis based on a random-effects model of data reported in 22 studies with 579 participants aged 3-25 year old. The results revealed an overall small to medium effect of exercise on cognition, supporting the efficacy of exercise interventions in enhancing certain aspects of cognitive performance in individuals with ASD and/or ADHD. Specifically, similar to the general population literature, the cognitive benefits of exercise are not consistent across all aspects of cognitive functions (i.e., some areas are not improved). The clinical significance of the reported effect sizes is also considered.
\end{abstract}

Keywords Meta-analysis - Exercise intervention ·

Cognition $\cdot$ ADHD $\cdot$ Autism

\section{Introduction}

The benefits of physical exercise have been widely recognised both in the literature (e.g., McMorris et al. 2009) and the media (Leavy et al. 2011; Marcus et al. 1998). Its reported positive effects can be broadly classified into physical health (e.g., WHO 2010), behavioural (e.g., Sowa and Meulenbroek 2012), cognitive (e.g., Kramer and Erickson 2007), and psychosocial health or functioning

Beron W. Z. Tan

b.tan@ecu.edu.au

1 School of Arts and Humanities, Edith Cowan University, 270 Joondalup Drive, Joondalup, WA 6027, Australia (e.g., Netz et al. 2005). One specific focus of research has been on the relationship between exercise and cognitive functions, ranging from studies of children (e.g., Tomporowski et al. 2008), to young adults (e.g., Lambourne and Tomporowski 2010), geriatric populations (e.g., Kramer and Erickson 2007), and non-clinical (e.g., McMorris and Hale 2012) and clinical populations (e.g., Eggermont et al. 2009).

Most of the research conducted concerns the general population and has reported mostly positive effects of exercise on cognitive performance, mainly on executive functions (e.g., Kramer and Erickson 2007; Tomporowski et al. 2008). Two key points have emerged from this research. Firstly, the effect of exercise on cognition is likely to be selective (e.g., Kramer and Erickson 2007; Tomporowski et al. 2008), even within executive functions (EF). Indeed, a recent meta-analysis by Verburgh et al. (2013), examined 24 studies $(\mathrm{N}=944)$ on the effects of acute and chronic exercise on domains of EF in healthy individuals aged 6-35 years. The authors reported a larger effect size on inhibition (i.e., $\mathrm{d}=0.46$ ), followed by planning (i.e., $\mathrm{d}=0.16$ ), and working memory (i.e., $\mathrm{d}=0.05$ ). However, the authors highlighted that the working memory and planning results and the effects of chronic exercise on executive functions should be interpreted with caution due to the small number of studies investigating these areas. Similarly, another meta-analysis of 79 studies involving healthy populations $(\mathrm{N}=2072)$ also found variability of exercise effects across various cognitive tasks reflecting different EF (Chang et al. 2012a). Secondly, the effect of exercise varies among individuals (e.g., Kramer and Erickson 2007; Tomporowski et al. 2008) and not every study reported cognitive improvements (e.g., Audiffren 2009; Kramer and Erickson 2007). For instance, it has been reported that individual 
differences such as fitness level moderate the magnitude of the effect of exercise on cognition; with greater improvements among those who are fitter (e.g., Chang et al. 2012a). Together, the two key points from the typical developing population literature has revealed the potential and limitations of using exercise in enhancing cognitive functions.

One of the implications provided by the literature from the typical developing population is that exercise may be particularly beneficial to individuals with learning difficulties (e.g., Fedewa and Ahn 2011; Sibley and Etnier 2003), such as those with attention deficit hyperactivity disorder (ADHD) or autism spectrum disorders (ASD). Unlike in the literature from the general population, however, the relationship between exercise and cognition in the neurodevelopmental population appears to be less clear, with studies on ASD samples focusing more on behavioural symptoms (e.g., Petrus et al. 2008; Sowa and Meulenbroek 2012) compared to ADHD studies that specifically examine EF (e.g., Chang et al. 2012b; Smith et al. 2013). This trend is not surprising as research in the general population regularly reveals specific improvements on EF following exercise (e.g., Tomporowski et al. 2008), and thus it has been theorised that exercise interventions may compensate for the impaired EF observed in individuals with ADHD (Wigal et al. 2013). Indeed, this hypothesis has been investigated in ADHD individuals but with inconclusive findings. For example, an earlier study conducted by Craft (1983), found that 1-10 min of stationary cycling did not produce cognitive benefits on working memory performance in 31 children with ADHD or in comparison with 31 healthy children. In partial support of this, Chang et al. (2012b) investigated the effects of running on a treadmill for $30 \mathrm{~min}$ on aspects of $\mathrm{EF}$ (i.e., inhibition and divided attention) in children with ADHD. Compared with participants in a sedentary control group $(\mathrm{n}=20)$, the exercise group $(\mathrm{n}=20)$ did not demonstrate greater EF performance, even though the authors reported post-exercise improvements from baseline scores in some aspects of EF tasks. In contrast to these studies though, Kang et al. (2011) reported improved cognitive performance (i.e., divided attention and working memory) in children with ADHD following a series of aerobic exercises $(\mathrm{n}=15)$ for $55 \mathrm{~min}$ compared to an educational control group $(\mathrm{n}=13)$. Despite conflicting findings, ADHD studies generally report positive findings of the effects of exercise interventions on aspects of EF (e.g., Choi et al. 2015; Pontifex et al. 2013) but the efficacy remains unclear.

A recent meta-analysis of eight studies reviewed the effects of exercise on various symptoms of ADHD children and provided the first indication of the magnitude of the effect on cognition (Cerrillo-Urbina et al. 2015). The authors reported moderate to large effect sizes (standardised mean differences of 0.58 and 0.84 ) on measures of EF and attention, respectively. However, although the meta-analysis detected various levels of risk for publication bias among the included articles, the analysis conducted could not account for the existing bias (e.g., reporting bias). Additionally, the reported positive effect of exercise on global EF was too broad. Therefore, it is unknown which aspects of EF are specifically impacted by exercise. This is important as it has been acknowledged in the general population literature that not all EF improves following exercise intervention (Tomporowski et al. 2008; Verburgh et al. 2013). Furthermore, as inhibition has been found to be commonly impaired in ADHD (e.g., Pennington and Ozonoff 1996), it is imperative to investigate whether this specific domain and other aspects of EF are influenced by exercise. Nonetheless, these limitations in the meta-analysis are likely due to the limited number of papers available and the focus being more on the general ADHD symptomology than cognition per se.

The ADHD literature appears to focus on positive findings of exercise intervention on EF. In particular, papers either reported a beneficial effect of exercise on global EF (Cerrillo-Urbina et al. 2015) or only statistically significant findings (e.g., Choi et al. 2015; Pontifex et al. 2013); and in those studies where non-improvements were acknowledged, this was attributed to the lack of task-sensitivity (e.g., Gapin et al. 2015; Pan et al. 2015). Although it is possible that the cognitive tasks used in the studies may be insensitive in detecting subtle cognitive changes, what is also possible or more likely based on the larger number of studies conducted on the general population, is that the effect of exercise is specific to some aspects of EF rather than to cognition in general and is evident in some individuals more than others (e.g., Tomporowski et al. 2008).

In terms of the ASD literature, what appears to be missing is an investigation of the effects of exercise on EF, as there have been suggestions that aspects of EF such as planning and set-shifting are impaired in ASD individuals (e.g., Pennington and Ozonoff 1996). To date, only one study has examined this issue. Anderson-Hanley et al. (2011) administered a single bout of exercise through the use of a gaming system (i.e., exergaming) for $20 \mathrm{~min}$ in 22 adolescents with ASD, to investigate the effect of exercise on aspects of EF and stereotyped behaviours. Compared with a sedentary control condition, post-exergaming participants demonstrated improvements in an EF task reflecting working memory but the results were less clear on set-shifting and inhibition. However, the authors did not report the findings of all the EF tasks involved in the study, further limiting the conclusions regarding the effect of exercise on $\mathrm{EF}$ in this population.

The gaps identified here in the literature may have important implications, particularly for the selection of appropriate interventions, as the symptoms of ASD and 
ADHD often do not appear in isolation (Gargaro et al. 2011; Joshi et al. 2014), even though non-comorbid cases also exist (e.g., Chantiluke et al., 2014). Furthermore, given that comorbidity between the two disorders is reported to be as high as over $80 \%$ of the cases (e.g., Joshi et al. 2010; Mukaddes et al. 2010), it is not known if exercise can be used as an effective intervention in improving aspects of cognitive functions in these clinical populations (i.e., comorbid and non-comorbid individuals). Although it is still contentious as to whether the two disorders are a common or unique subtype that falls under the domain of either ASD or ADHD exclusively (e.g., Chantiluke et al. 2014), there is a general agreement that symptom overlap exists even if individuals may not fulfil the diagnostic criteria of both disorders. Therefore, it has been recommended that the uniqueness and co-morbid characteristics between both disorders be acknowledged (Gargaro et al. 2011). This review attempts to study the efficacy of exercise intervention on cognition in both disorders, concurrently and separately.

In terms of executive dysfunction, some specific EF domains are generally reported to be impaired in individuals with ASD and ADHD, when compared separately to healthy individuals. In ASD individuals, EF domains including aspects of planning (e.g., Chen et al. 2016; Hill 2004), set-shifting and working memory (e.g., Chen et al. 2016; Andersen et al. 2015) are usually impaired. Conversely, deficits are commonly found in aspects of inhibition (e.g., Willcutt et al. 2005) and working memory (e.g., Schreiber et al. 2014) in individuals with ADHD. There are also findings that EF in general tend to be more impaired in individuals with ASD than those with ADHD (e.g., Corbett et al. 2009; Goldberg et al. 2005; Pennington and Ozonoff 1996). It is noteworthy that there are inconsistencies within ASD and ADHD literature on which aspects of EF in these individuals are impaired or intact, which is beyond the scope of this review. Nevertheless, studies that investigated EF between individuals with ASD and ADHD have demonstrated the difficulty in establishing a distinct executive dysfunction profile (e.g., Corbett et al. 2009; Geurts et al. 2004; Goldberg et al. 2005); in that EF deficits can sometimes overlap in both disorders such as aspects of working memory, sustained attention and even inhibition. Considering that the literature in the healthy population generally reported exercise benefits on EF (e.g., Kramer and Erickson 2007; Tomporowski et al. 2008), especially on inhibition and probably other aspects of EF (Verburgh et al. 2013), exercise may have the potential to be used as an intervention that targets EF deficits in individuals with ASD and/or ADHD.

In summary, the existing literature has provided valuable information on the effects of exercise on cognition in individuals with ASD/ADHD. Nevertheless, what seems to be lacking is also a practical interpretation of effect size (Ellis 2010) that would be useful for clinicians and parents. The purpose of this review is to investigate the efficacy of exercise intervention on individuals with ASD/ADHD, and explore the practical significance of applying exercise to cognition based on the meta-analytic findings. To the authors' knowledge, this is the first meta-analysis that reviews the relationship between exercise and cognition, in both ASD and ADHD populations.

\section{Methods}

\section{Inclusion Criteria}

For inclusion in this meta-analysis, the participants of a study were required to have been diagnosed with ADHD or ASD, including disorders previously known as autism, Asperger's and pervasive developmental disorders not otherwise specified (American Psychiatric Association 2013). Studies were required to have used exercise as an intervention in evaluating some aspects of objective cognitive performance (excluding self-report measures). In addition, papers were required to be from quantitative studies published in journals or dissertations from 1968 to 2015 .

\section{Review Process (January-June 2015)}

The process of this review was based on the methods highlighted in Field and Gillett (2010). A systematic search of the literature using a series of keywords was conducted in various databases: PsychINFO, CINAHL, PubMED, Web of Science and ERIC. Keywords such as "physical activit*", "exercis*", 'cogniti* improv*', "mental function*", "performance", "autis*", "autism spectrum disorder*", "asperger*", "pervasive developmental", "ADHD", "ADD", "attention-deficit disorder*" and "attention-deficit hyperactivity disorder*", and search connectors like "AND", "OR" and "NOT" were used in the search process. Articles were assessed based on their relevance to the purpose and inclusion criteria of this review on three levels: title, abstract and content. Articles that were retained by the content level were used for a handsearch of their reference lists to check for articles that may have been missed in the database search. In addition, a forward-search of the final selected articles on Google scholar was conducted to locate articles that cited the selected articles. Lastly, relevant journals that were not within the aforementioned databases were also searched for potential articles. This series of search processes ensured relevant papers in the literature were identified and included in the meta-analysis. Consensus among the authors as 
to those articles selected for the analysis was achieved and any disagreement was resolved through discussion. The initial search across the aforementioned databases produced 32,767 titles, of which 83 papers were selected in the abstract phase and 17 papers for content evaluation; this series of processes led to 10 papers fulfilling the inclusion criteria. This was then followed by a backward and forward search of the included articles which further revealed another 9 papers for inclusion. Additionally, other sources from journals and websites generated 5 more articles, increasing the number of papers that fulfilled the inclusion criteria to 24 . However, two articles only published their abstracts and the respective authors were contacted to obtain the full articles, but this was unsuccessful. Overall, 22 articles were included in the meta-analysis.

\section{Process of Calculating Effect Sizes}

The mean differences between the experimental group and the control group/baseline conditions provided by the included articles $(\mathrm{N}=22)$ were used to calculate the effect sizes as measured by Glass's delta $(d)$; where the mean differences were standardised using the standard deviations of the control group/condition (Field and Gillett 2010). The obtained values for effect sizes $d$ were then converted into Pearson's correlation coefficient, $r$. In cases where the means and standard deviations were not provided, the $\mathrm{F}$ or $\mathrm{t}$ statistics, and odds ratio were used to convert the relevant metrics into effect size $r$. All effect size calculations and conversions were based on formulas reported in Field and Gillett (2010), Borenstein et al. (2009), and Wolf (1986), and were estimated up to nine decimal places for accuracy (Field 2005). It is noteworthy that in some instances, the calculated effect size differed from what was reported in the respective articles. This difference was due to the choice of comparisons that respective papers were based upon. For example, some studies computed within-group effect sizes rather than comparing between treatment and control groups when available (e.g., Chang et al. 2012b). The direction of the effect was computed such that positive effect sizes indicated improvements while negative effect sizes represented attenuation in cognitive performance after exercise intervention. To prevent inflating the estimated population effect size by studies that provided multiple effect sizes, only an average effect size per article was entered into the meta-analysis (Rosenthal 1991). Furthermore, each effect size was transformed using Fisher's (1921) $r$-to- $z$ conversion before running the meta-analysis using a random-effects model described by Hedges and Vevea (1998), and the obtained overall $\mathrm{Zr}$ was transformed back to $r$ for interpretation. A random-effects model was selected as it was recommended that variability in effect sizes should be assumed in psychological research and that this model allowed generalisation of the findings beyond the studies included in the meta-analysis (Field and Gillett 2010).

To examine potential variables that might account for the efficacy of exercise on cognition, a multiple regression model was used, with eight moderator variables that were either categorical (diagnosis, age, control type, cognitive tasks, exercise type, and single/multiple exercise sessions) or continuous (exercise duration and sample size). Sensitivity analysis (Vevea and Woods 2005) was also conducted to evaluate and account for the level of publication bias. All statistical analyses were based on SPSS and R syntax provided by Field and Gillett (2010). Lastly, binomial effect size display (BESD) was calculated to assist in the practical interpretation of effect size based on the formula reported in Randolph and Edmondson (2005).

\section{Results}

\section{Descriptive Results}

This meta-analysis included 22 articles (16 ADHD, 6 ASD) with a total sample size of 579 participants $(\mathrm{ADHD}=451$; $\mathrm{ASD}=128$ ) aged from 3 to 25 years. The gender distribution was 416 males to 48 females, with the gender of 115 participants unclear or not reported. Tables 1, 2, 3 show the stem-and-leaf plots of all effect sizes for combined ASD/ ADHD studies, and separated by diagnosis. Overall, the mean effect size was computed from 125 effect size values.

\section{Main Findings}

Based on Hedges and Vevea's (1998) random-effects model, the overall mean effect size is $r=.235$, with a $95 \%$ confidence interval between .131 and .335 , and a significant $z$-score, $z=4.347, p<.001$. The overall mean effect size shows a small to moderate magnitude of effects of exercise intervention on aspects of cognition in

Table 1 Stem-and-leaf plot of overall effect sizes for ASD/ ADHD $(\mathrm{N}=22)$

\begin{tabular}{ll}
\hline Stem & Leaf \\
\hline-.0 & 4,7 \\
.0 & $0,0,1$ \\
.1 & $1,1,2,4,5,6,8,8$ \\
.2 & 0 \\
.3 & 0,8 \\
.4 & 2,5 \\
.5 & $3,3,8$ \\
.6 & 2 \\
\hline Values estimated to 2 decimal \\
places
\end{tabular}



effect sizes for ADHD studies $(\mathrm{n}=16)$

Table 3 Stem-and-leaf plot of effect sizes for ASD studies $(\mathrm{n}=6)$
Table 2 Stem-and-leaf plot of

\begin{tabular}{ll}
\hline Stem & Leaf \\
\hline-.0 & 4,7 \\
.0 & $0,0,1$ \\
.1 & $1,1,2,8,8$ \\
.2 & 0 \\
.3 & 0,8 \\
.4 & 2 \\
.5 & 3,8 \\
\hline Values estimated to 2 decimal \\
places
\end{tabular}

\begin{tabular}{ll}
\hline Stem & Leaf \\
\hline .0 & \\
.1 & $4,5,6$ \\
.2 & \\
.3 & \\
.4 & 5 \\
.5 & 3 \\
.6 & 2 \\
\hline
\end{tabular}

Values estimated to 2 decimal places

individuals with ADHD and ASD. The homogeneity test, as denoted by the $\mathrm{Q}$ statistic is not significant, $\chi^{2}$ $(21)=29.08, p=.11$ but the $I^{2}=27.78 \%$, suggests that there is a low to medium heterogeneity within the sample of effect sizes included in this analysis. This is evident from the forest plot in Fig. 1, which reveals the various effect size values for physical exercise on cognition reported in the studies included in this meta-analysis with $95 \%$ confidence intervals. The dotted line in the middle represents the line of no effect. The figure identified that only 3 out of 22 papers had their confidence intervals within the range of positive effect size values, with the majority of the papers having confidence intervals extending into the range of negative values. Lower confidence intervals in the negative range may in part be due to the limited sample sizes used in some of the studies resulting in low power for detecting a small effect, as revealed by the overall effect size estimate. Nevertheless, the mean effect size estimates for ASD, ADHD and combined samples have confidence intervals supporting a positive effect of exercise intervention. These results indicate that the positive effects of exercise on cognition are likely to exist in these populations but the exact magnitude could not be confidently established. Taken together, the use of a random-effects model is appropriate and a moderator analysis could be conducted to explain the variability. Table 4 shows the results of the meta-analyses for ASD and ADHD studies combined, and separately.

\section{Publication Bias and Sensitivity Analysis}

Rosenthal's (1979) fail-safe $\mathrm{N}$ analysis reveals 186 unpublished papers (i.e., null effect) are needed to be included in the meta-analysis in order to invalidate the population effect size of small to medium (i.e., .235) found in this review. Furthermore, Begg and Mazumdar's (1994) rank correlation test shows a non-significant correlation between the effect sizes and sample size of the studies included in the meta-analysis, $\tau(\mathrm{N}=22)=.11, p=.46$. Although the result of Kendall's tau is non-significant, in view of the small number of studies included in the metaanalysis, publication bias cannot be rejected and therefore a sensitivity analysis (Vevea and Woods, 2005) was conducted to estimate and account for the likelihood of publication bias. When applied under a severe selection (i.e., one-tailed) model, the population effect size reduced from .235 to -.466 ; this suggests a lower level of confidence is appropriate in the effects of exercise on cognition in this neurodevelopmental population. Sensitivity analyses were then conducted separately for ASD and ADHD samples to further understand the data. For the ASD sample, the adjusted population effect size does not result in much attenuation (i.e., .471 reduced to .439 ), indicating that the population effect size remains relatively unaffected by the most severe scenario for one or two-tailed selection bias. In contrast to this, the adjusted population effect size for the ADHD sample is severely impacted by a one-tailed selection bias, decreasing the effect size from .181 to -.452 , suggesting that the actual effect size estimate for this population may be smaller than the mean effect size. Therefore, it is likely that the overall sensitivity results for both populations are affected by the effect size values from the ADHD sample. One possible explanation is the issue of reporting bias; 4 out of the 16 ADHD articles did not report the results of any non-significant findings (i.e., Choi et al. 2015; Gawrilow et al. 2016; Pontifex et al. 2013; Ziereis and Jansen 2015). Another factor may be the tasks involved in evaluating aspects of cognition, which is addressed in the next section.

\section{Moderator Analysis}

Based on the moderator analysis (see Table 5), only the type of cognitive tasks significantly moderated the population effect size, $\chi^{2}(1)=4.08, p=.04$. Indeed, the majority of the ASD studies evaluated on-task duration or simple learning tasks $(n=5)$, except for Anderson-Hanley et al. (2011), which examined executive functions. Conversely, all ADHD studies $(n=16)$ investigated aspects of executive functioning. Regardless of diagnosis, individual meta-analysis by cognitive tasks demonstrated larger population effect size values for on-task duration/simple 
Fig. 1 A forest plot displaying the effect sizes and confidence intervals of studies included in the meta-analysis. Note All effect sizes are corrected to 3 decimal places. Mean effect sizes for ASD and/or ADHD are based on the random-effects model. Open square box ASD studies. Filled square box ADHD studies

\section{5\% Confidence Intervals of all Effect Sizes}

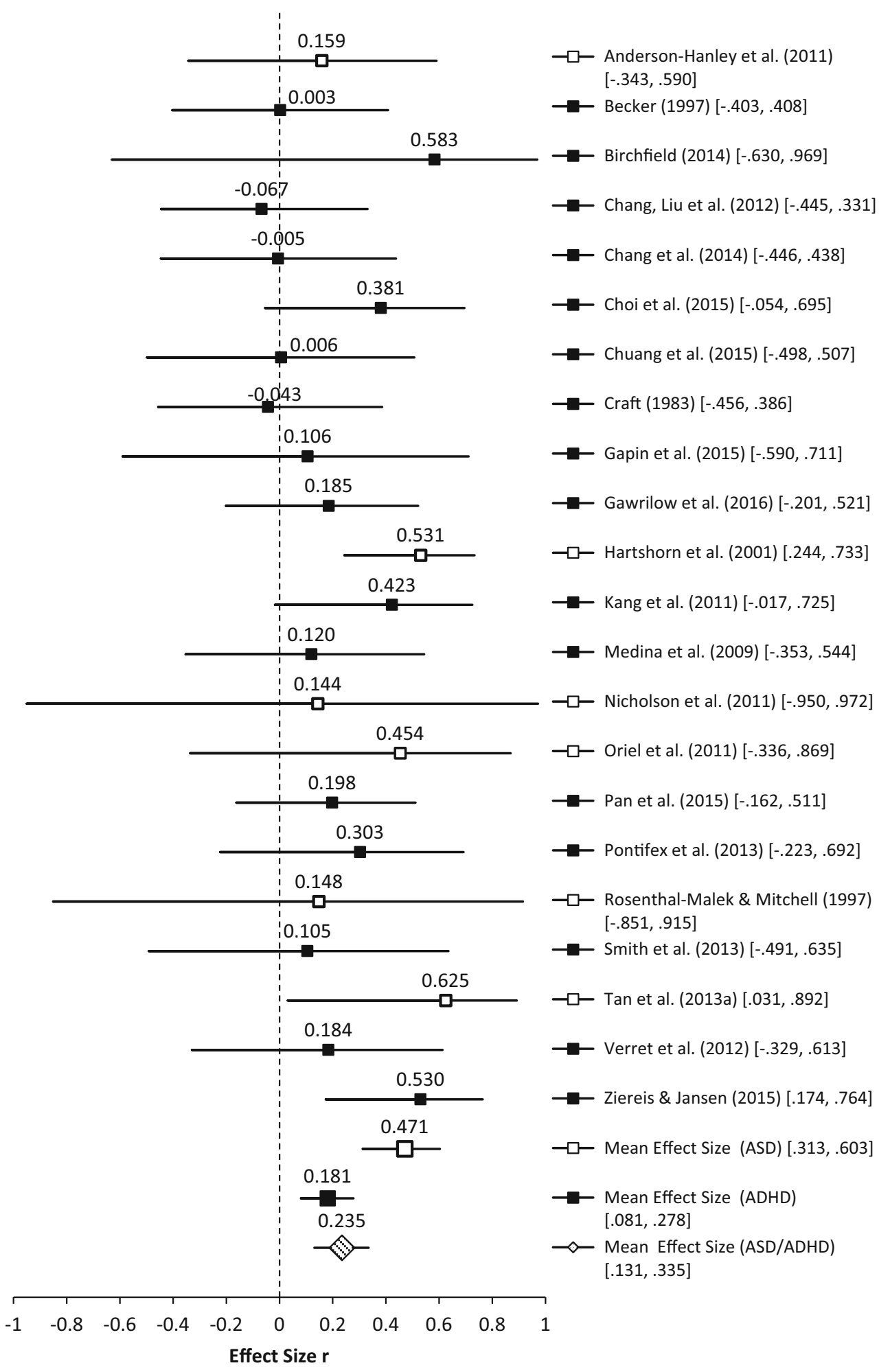

learning tasks $(\mathrm{n}=5), r=.526(95 \%$ confidence inter$\mathrm{val}=.362, .658)$, than for $\mathrm{EF}$ tasks $(\mathrm{n}=17), r=.180$ (95\% confidence interval $=.087,271)$. As EF is a broad umbrella term that involves functions such as inhibition, set shifting, planning and working memory (Pennington and Ozonoff 1996), the efficacy of exercise would be better understood by analysing its cognitive effect with respect to EF domains. 
Table 4 Results of individual random-effects meta-analyses by diagnostic group
Table 5 Moderators effect on the efficacy of exercise on cognition in ASD and ADHD samples

\begin{tabular}{lrllllllll}
\hline Group & \multicolumn{7}{l}{$95 \%$ confidence intervals } \\
\cline { 2 - 9 } & \multicolumn{1}{c}{$k$} & $n$ & $\tau$ & $\mathrm{Q}$ & Lower & Mean ES & Upper & $z$ & $p$ \\
\hline ASD/ADHD & 22 & 579 & .017 & 18.02 & .131 & .235 & .335 & 4.35 & $<.01^{*}$ \\
ASD & 6 & 128 & .000 & 3.65 & .313 & .471 & .603 & 5.36 & $<.01^{*}$ \\
ADHD & 16 & 451 & .003 & 14.76 & .081 & .181 & .278 & 3.51 & $<.01^{*}$ \\
\hline
\end{tabular}

$k$ number of studies, $n$ sample size

$* \mathrm{p}<.05$

\begin{tabular}{|c|c|c|c|c|c|}
\hline \multirow[t]{2}{*}{ Continuous predictors } & \multicolumn{5}{|c|}{$95 \%$ confidence intervals for $b$} \\
\hline & Lower & Point estimate & Upper & $t$ & $p$ \\
\hline Duration of exercise & -.014 & -.004 & .005 & -.995 & .34 \\
\hline Sample size & -.008 & -.001 & .006 & -.348 & .74 \\
\hline Categorical Predictors & \multicolumn{2}{|c|}{ Comparisons } & $\chi^{2}$ & $d f$ & $p$ \\
\hline Diagnosis & \multicolumn{2}{|c|}{$\begin{array}{l}\text { ASD } \\
\text { ADHD }\end{array}$} & 1.73 & 1 & .19 \\
\hline Exercise type $^{\mathrm{a}}$ & \multicolumn{2}{|c|}{$\begin{array}{l}\text { Single activity } \\
\text { Mixed activity }\end{array}$} & 3.37 & 1 & .07 \\
\hline Age (Years) & $\begin{array}{l}3-10 \\
11-1 \\
19-2\end{array}$ & & 0.77 & 2 & .68 \\
\hline Control & \multicolumn{2}{|c|}{ Control group } & 0.38 & 1 & .54 \\
\hline Cognitive tasks & \multicolumn{2}{|c|}{$\begin{array}{l}\text { On-task duration/Learning } \\
\text { EF tasks }\end{array}$} & 4.08 & 1 & $.04 *$ \\
\hline Session number & \multicolumn{2}{|c|}{ Single bout } & 0.14 & 1 & .71 \\
\hline
\end{tabular}

$\mathrm{N}=22$ studies

$* \mathrm{p}<.05$

${ }^{\text {a }}$ All exercise interventions involved some form of aerobic exercises; single activity refers to any type of aerobic exercise that involved only one-type of physical activity (e.g., treadmill), while mixed activity represents a mixture of various kinds of aerobic and perceptual-motor exercises

\section{Meta-Analysis by EF Domains}

As there is only one ASD paper that included EF tasks (i.e., Anderson-Hanley et al. 2011), it is not included in the metaanalysis by EF domains. The EF tasks from the 16 ADHD articles are categorised into inhibition $(n=11)$, memory $(\mathrm{n}=6)$, and set shifting $(\mathrm{n}=5)$. Only inhibition and memory functions demonstrated significant small to medium ES following post-exercise intervention, $r=.181$ and $r=.286$, respectively (see Table 6). Sensitivity analysis revealed a reduced effect size on inhibitory control for severe one-tailed selection bias, $r=.097$, and memory function, $r=-.500$. This result means that the estimated effect sizes for both EF domains may be smaller than the findings of the individual meta-analysis. Interestingly, the attenuated effect size for memory function reduced drastically and reversed the positive effect of exercise (i.e., exercise has a negative impact on memory functions). However, the finding on memory should be interpreted with caution as the number of papers included is limited $(n=6)$. Moreover, the memory tasks included a range of memory functions (e.g., visual/verbal working memory), and thus it is also possible that the exercise effect varies for different aspects of memory (Chang et al. 2012a).

\section{Discussion}

\section{Efficacy of Exercise Interventions on Individuals with ASD and/or ADHD}

The results from this meta-analysis support the efficacy of exercise on improving some aspects of cognition in young individuals with ASD and/or ADHD. The overall effect of exercise intervention has a small to medium effect on ASD/ ADHD individuals, $r=.235$. This is similar to the overall 
Table 6 Results of individual random-effects meta-analyses by executive functions (ADHD)

\begin{tabular}{lccccccccc}
\hline Group & \multicolumn{7}{l}{$95 \%$ confidence intervals } \\
\cline { 2 - 10 } & $k$ & $n$ & $\tau$ & $\mathrm{Q}$ & Lower & $r$ & Upper & $z$ & $p$ \\
\hline Executive functions & 16 & 451 & .003 & 14.76 & .081 & .181 & .278 & 3.51 & $<.01^{*}$ \\
Inhibition & 11 & 288 & .000 & 3.95 & .057 & .174 & .287 & 2.89 & $<.01^{*}$ \\
Memory & 6 & 168 & .043 & 4.21 & .027 & .286 & .510 & 2.16 & $.03^{*}$ \\
Set shifting & 5 & 127 & .023 & 3.83 & -.029 & .184 & .382 & 1.70 & .09 \\
\hline
\end{tabular}

$k$ number of studies, $n$ sample size

$* \mathrm{p}<.05$ effect size estimate reported ( $d=0.32$, equivalent to $r=.16)$ in children with/without learning or physical disabilities (Fedewa and Ahn 2011; Sibley and Etnier 2003), but closer to the effect size found $(d=0.52$, equivalent to $r=.25)$ in healthy individuals aged 6-35 years (Verburgh et al. 2013). Given that this metaanalysis combines ASD and ADHD samples, diagnosis is expected to be a potential moderator on the overall effect size. Contrary to expectation, though, diagnosis was not a significant moderator. Although this may be due to the small number of ASD studies $(n=6)$, it may also be due to high-comorbidity shared between both disorders (e.g., Joshi et al. 2010). This finding indicates that exercise interventions are likely to be beneficial to both ASD and ADHD populations. Similarly, sample size, age group, the type of control group/condition, and the type of exercise and number or duration of intervention sessions did not significantly moderate the relationship between exercise and cognition. These findings are partially consistent with other meta-analyses. Sibley and Etnier (2003) also reported that the effect of exercise is not dependent on the type of exercises but Fedewa and Ahn's (2011) meta-analysis found larger positive effect of aerobic exercise compared with other types of exercise interventions (e.g., perceptualmotor exercises). Given that the present review included studies that conducted exercise interventions that involved either aerobic-type or aerobic and perceptual-motor exercises, the current finding did not find any significant differences in their impact on cognitive functions. In addition, other meta-analyses found a greater post-exercise cognitive benefit in younger children (Sibley and Etnier 2003; Fedewa and Ahn 2011), contrary to this finding where the exercise-cognition effect was more likely to be beneficial across ASD/ADHD individuals aged between 3 and 25 years; similar to findings from the general population reported by Verburgh et al. (2013). Despite methodological differences in the type of populations (i.e., clinical versus general population) included in the various meta-analyses, a consistent finding is that the relationship between exercise and cognition is moderated by the type of cognitive tasks (e.g., Chang et al. 2012a; Fedewa and Ahn 2011; Sibley and Etnier 2003; Verburgh et al. 2013).

\section{Efficacy of Exercise Interventions by Cognitive Tasks}

The effects of exercise interventions on cognition differ based on the type of cognitive tasks used to evaluate its efficacy. In the current review, the cognitive tasks were separated into two broad categories, on-task duration/simple learning tasks (i.e., the length of time individuals stayed engaged on a specific task; or for example, the number of correct responses on the value of various coins presented, see Rosenthal-Malek and Mitchell 1997) and global executive functions. Current findings demonstrated that exercise interventions have a larger effect on the duration spent on-task and the completion of simple tasks than EF tasks. When diagnosis type by cognitive tasks are considered, exercise has a large effect on on-task duration/simple tasks in individuals with ASD, $r=.526(\mathrm{n}=5)$. With regards to ADHD samples $(\mathrm{n}=16)$, exercise has a small to medium effect on EF, $r=.181$. This is a smaller effect compared to the effect size reported by Cerrillo-Urbina et al. (2015), $d=0.58$ (equivalent to $r=.28$ ), possibly due to a smaller number of articles included in their analysis $(\mathrm{n}=3)$. Furthermore, this review extended the findings of CerrilloUrbina et al. (2015) by including the original 3 articles in their paper that examined EF, further reinforcing the conclusion that exercise interventions have positive effects on $\mathrm{EF}$ in ADHD individuals.

\section{Efficacy of Exercise Interventions on Executive Functions (ADHD)}

The meta-analysis indicates that exercise interventions have specific beneficial effects on inhibitory and memory functions ( $r=.174$ and $r=.286$, respectively), but there are unclear effects on set shifting performance. Even under the most severe one-tailed selection bias model, exercise interventions remain effective in enhancing inhibition in 
ADHD individuals (i.e., $r=.097$ ). This indicates that exercise interventions could be particularly useful for targeting improvement in inhibition aspects of EF. On the contrary, memory benefits after exercise may exist but the effect may be much smaller, and varies among different types of memory functions. For instance, another metaanalytic study reported that visual short-term memory improves following exercise, $d=0.49$ (equivalent to $r=.24$ ), but exercise has a negative impact on other aspects of memory including sequential and auditory-verbal memory (Chang et al. 2012a). Overall, the present meta-analysis partially supports the hypothesis that exercise may be essential in compensating for the EF deficits, especially inhibitory control observed in this clinical population (Wigal et al. 2013).

\section{Individual Variations}

Based on the summary table ("Appendix"), it is apparent that there is a wide range of cognitive tasks that measures various aspects of EF, and most importantly, not all functions were improved by exercise interventions. This finding is similar to those studies based on the general population (e.g., Kramer and Erickson 2007; Tomporowski et al. 2008). Further, the effect of exercise is selective and affects some areas of cognition more than others. If exercise is to be treated as a form of "medication", it is also necessary to acknowledge its limitations. This will facilitate advancement in the science of using exercise to improve cognition by identifying what areas of cognition are not affected by exercise, and which population or factors limit its effectiveness (e.g., fitness level, diagnosis). Specifically, why does person A improve but not person B? The focus on "why" will bring the field a step closer to uncovering the mechanism of exercise on cognition.

\section{Limitations/Future Directions}

The present meta-analysis bridges the gap in the literature regarding the exercise and cognition relationship in the ASD/ADHD and typical developing populations. Although the overall findings are encouraging, it should be regarded as a tentative conclusion in guiding future research and future large-scale randomised-controlled trials would be required to validate the current findings. Moreover, the findings should also be interpreted in the context of the existing limitations. Firstly, the number of articles available and included in this review (i.e., 22 studies) is still considered relatively small compared to other meta-analyses in the typical developing population literature. In particular, the number of ASD studies examining exercisecognition relationship is very limited (i.e., six studies), especially on EF. Secondly, five studies (one ASD and four ADHD articles) did not report non-significant results. This reporting bias is consistent with the results of the sensitivity analysis. As mentioned earlier regarding the limitations of exercise interventions, future studies should try to include at least some basic information on non-significant findings (e.g., effect size, means, and standard deviations). Furthermore, similar to drug trials, it would be informative to also report the number of participants that show improvements in their cognition. This is because most studies in the literature only rely on the mean statistics and it may be possible that the number of individuals that truly improve in cognition after exercise interventions is low but the mean score of the experimental group/condition is high or significantly different to the control group/condition (see Speelman and McGann 2013 regarding limitations of the mean). Thirdly, variability in individual differences such as developmental and fitness levels, and the intensity of exercise interventions are identified in the literature as important moderators in understanding the exercise-cognition relationship (e.g., Chang et al. 2012a; Kramer and Erickson 2007; Tomporowski et al. 2008); however, 18 studies did not report IQ levels and 12 studies did not provide quantification of their exercise interventions (e.g., heart rate, oxygen consumption). Therefore, these factors could not be examined in this meta-analysis. Fourthly, the current results could only support the use of exercise in enhancing aspects of cognition for individuals up to 25 years of age. Therefore, it is unknown if exercise interventions are equally effective in older age groups with ASD/ADHD. Lastly, another limitation is the inherent issues within EF tasks. Due to the complex nature of EF, an EF task is usually unable to provide an isolated measure of a specific cognitive process that it intends to measure and invariably also captures other aspects of EF and non-EF processes (e.g., Pennington and Ozonoff 1996; Suchy 2009). Moreover, most studies included in the meta-analysis examined each EF domain with a single neuropsychological task, making it difficult to ascertain whether an improvement (or not) on a single task after exercise intervention is indeed a reflection of an actual change in that particular EF. In addition, recent literature has supported EF as a multifaceted latent construct where various domains are separable but interdependent (e.g., Cassidy 2016). Thus, future experimental studies could consider using a number of neuropsychological tasks to assess each EF domain (Ziereis and Jansen 2015) and if possible, to include a range of $\mathrm{EF}$ measures (see e.g., Smith et al. 2013). Although the present meta-analysis is unable to evaluate the exercise effect on other EF such as planning, sustained attention and working memory due to limited number of papers examining these areas; nevertheless, various neuropsychological tasks used in the studies (refer 
Table 7 Binomial effect size display (BESD): efficacy of post-exercise interventions on cognition

\begin{tabular}{lllll}
\hline Condition & Effect size $(r)$ & \% Improved & \% Not improved & Variance $\left(r^{2}\right)$ \\
\hline ASD/ADHD & .235 & 61.75 & 38.25 & .06 \\
ASD (on-task/learning task) & .526 & 76.30 & 23.70 & .28 \\
ADHD (executive function) & .181 & 59.05 & 40.95 & .03 \\
ADHD (inhibition) & .174 & 58.70 & 41.30 & .03 \\
ADHD (memory) & .286 & 64.30 & 35.70 & .08 \\
\hline
\end{tabular}

to "Appendix") are combined to evaluate the effect of exercise intervention on inhibition, set-shifting and memory functions (including working memory). Specifically, there is support that exercise benefits inhibitory function in individuals with ADHD.

\section{Clinical Implications: Binomial Effect Size Display}

A major issue that has been overlooked in the literature is the practical interpretation of what effect size actually means in the context of applying exercise interventions in improving cognitive functions. The binomial effect size display (BESD) by Rosenthal and Rubin (1982) enables the interpretation of effect size in meaningful terms: the estimated percentage of individuals that improved aspects of their cognition by exercise interventions. Notwithstanding the limitations, the conversion of overall $r$ to BESD (Table 7) demonstrates that relative to control groups or baseline measures, overall, following exercise interventions, $61.75 \%$ of the individuals with ASD and ADHD improved on aspects of their cognitive performance. Specifically, exercise benefited $76.30 \%$ of ASD individuals by enhancing their on-task behaviour and performance on simple learning task. For ADHD individuals, 59.05\% reported cognitive benefits on aspects of their EF after exercising; especially on inhibitory control (i.e., 58.70\%). Based on Table 7, it is evident that exercise interventions mostly only account for a small variance on cognitive improvements, supporting the consensus in the literature that the relationship between exercise and cognition is complex and is moderated by many other factors such as individual differences (e.g., Chang et al. 2012a; Tomporowski et al. 2008). Nonetheless, it should be noted that the implications of utilising exercise interventions with individuals with ASD and/or ADHD should not be underestimated, as it is likely to be particularly useful in addressing areas of their cognitive function in which they typically do not perform well (e.g., inhibition). Furthermore, apart from exercise-induced improvement in aspects of $\mathrm{EF}$, which is likely to be related to neurobiological pathways such as catecholamine (see e.g., Wigal et al. 2013), neurotrophic and growth factors (see e.g., Ratey and Loehr 2011); indirect pathways including elevated self- efficacy, mood, and other psychosocial functioning (e.g., Davis and Lambourne 2009; Tan et al. 2013b; Tomporowski et al. 2011) may also be beneficial to individuals with ASD and/or ADHD.

\section{Conclusion}

The findings from this meta-analysis support the efficacy of using exercise interventions in improving some aspects of cognitive functions in individuals with ASD and/or ADHD between the ages of 3-25 years old. Additionally, similar to conclusions from the typical developing literature, the exercise effect varies among individuals and favours some cognitive functions over others. Although the mechanism behind the unique characteristics of the exercise effect on cognition remains inconclusive, acknowledging the limitations of what exercise can and cannot do provides important groundwork towards further understanding the relationship between exercise and cognition.

Author's contributions BT designed the study and wrote the protocol. All authors participated in the literature search process and identified relevant articles for inclusion in the meta-analysis. BT coded the articles and conducted the statistical analysis. JAP and CS provided statistical guidance. BT wrote the first draft of the manuscript and all authors contributed to and have approved the final manuscript.

\section{Compliance with Ethical Standards}

Conflict of interests Beron Tan declares that he has no conflict of interest. Julie Ann Pooley declares that she has no conflict of interest. Craig Speelman declares that he has no conflict of interest.

Ethical approval This article does not contain any studies with human participants or animals performed by any of the authors.

Open Access This article is distributed under the terms of the Creative Commons Attribution 4.0 International License (http://creative commons.org/licenses/by/4.0/), which permits unrestricted use, distribution, and reproduction in any medium, provided you give appropriate credit to the original author(s) and the source, provide a link to the Creative Commons license, and indicate if changes were made.

\section{Appendix}

\section{See Table 8 .}




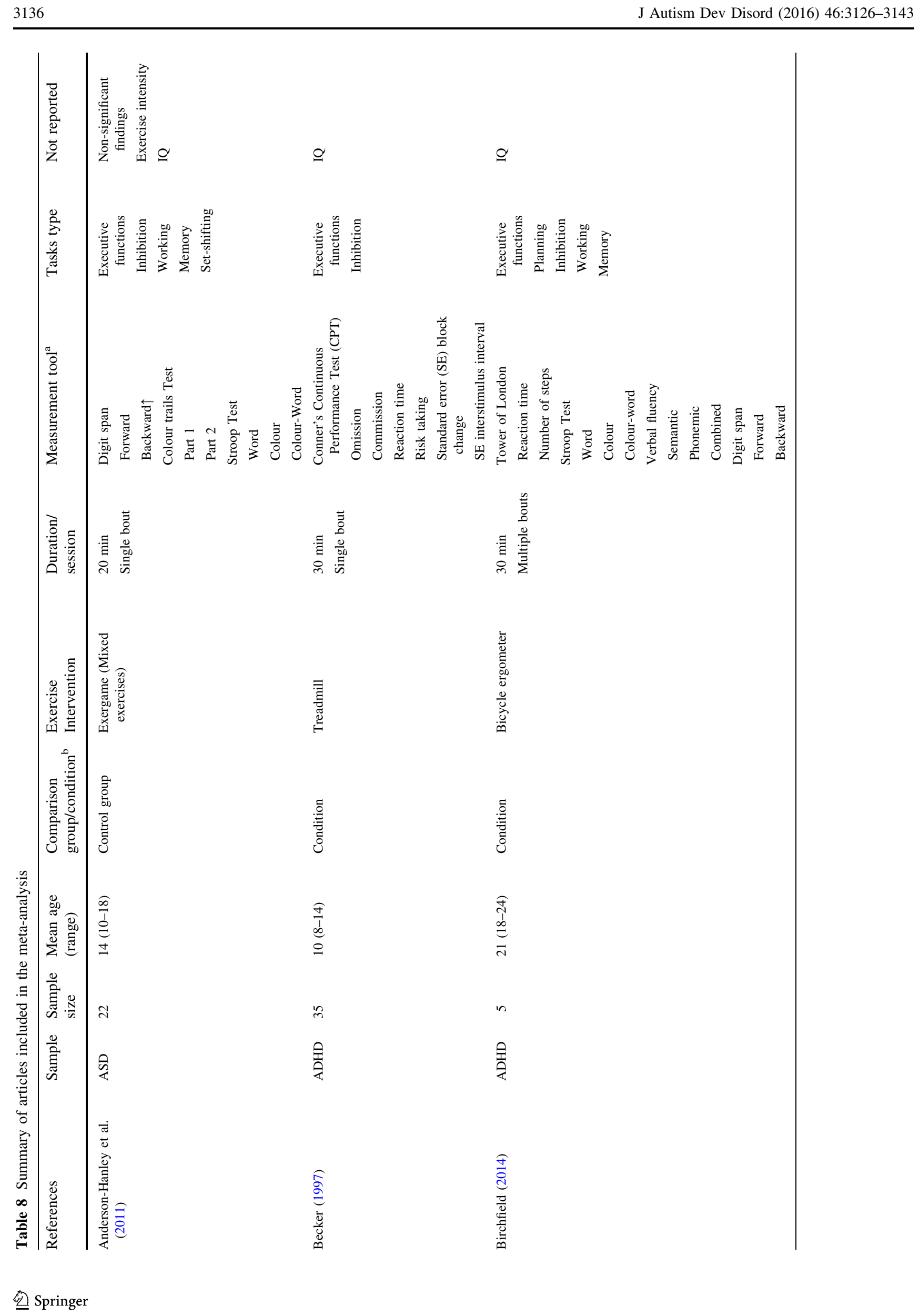




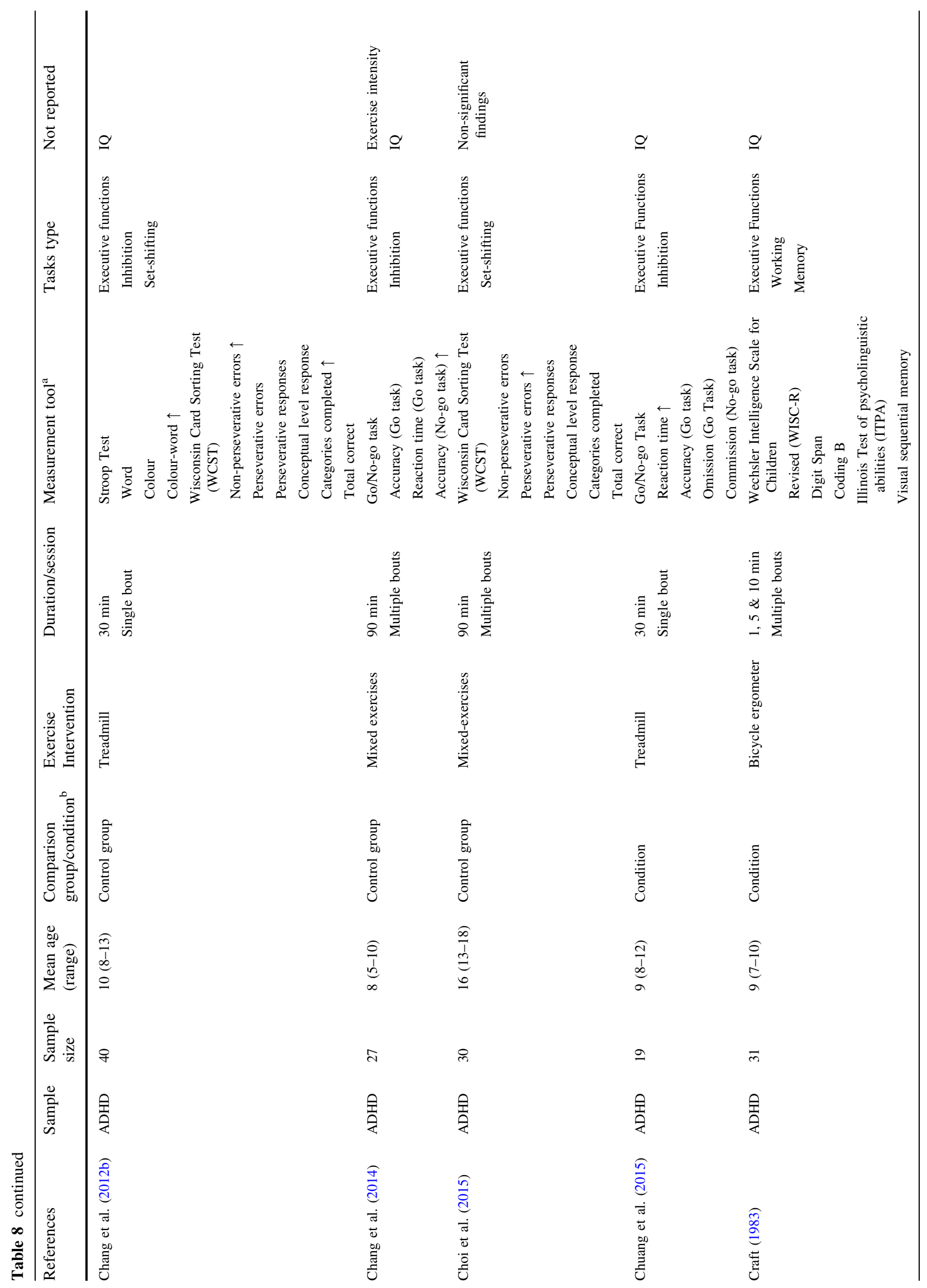




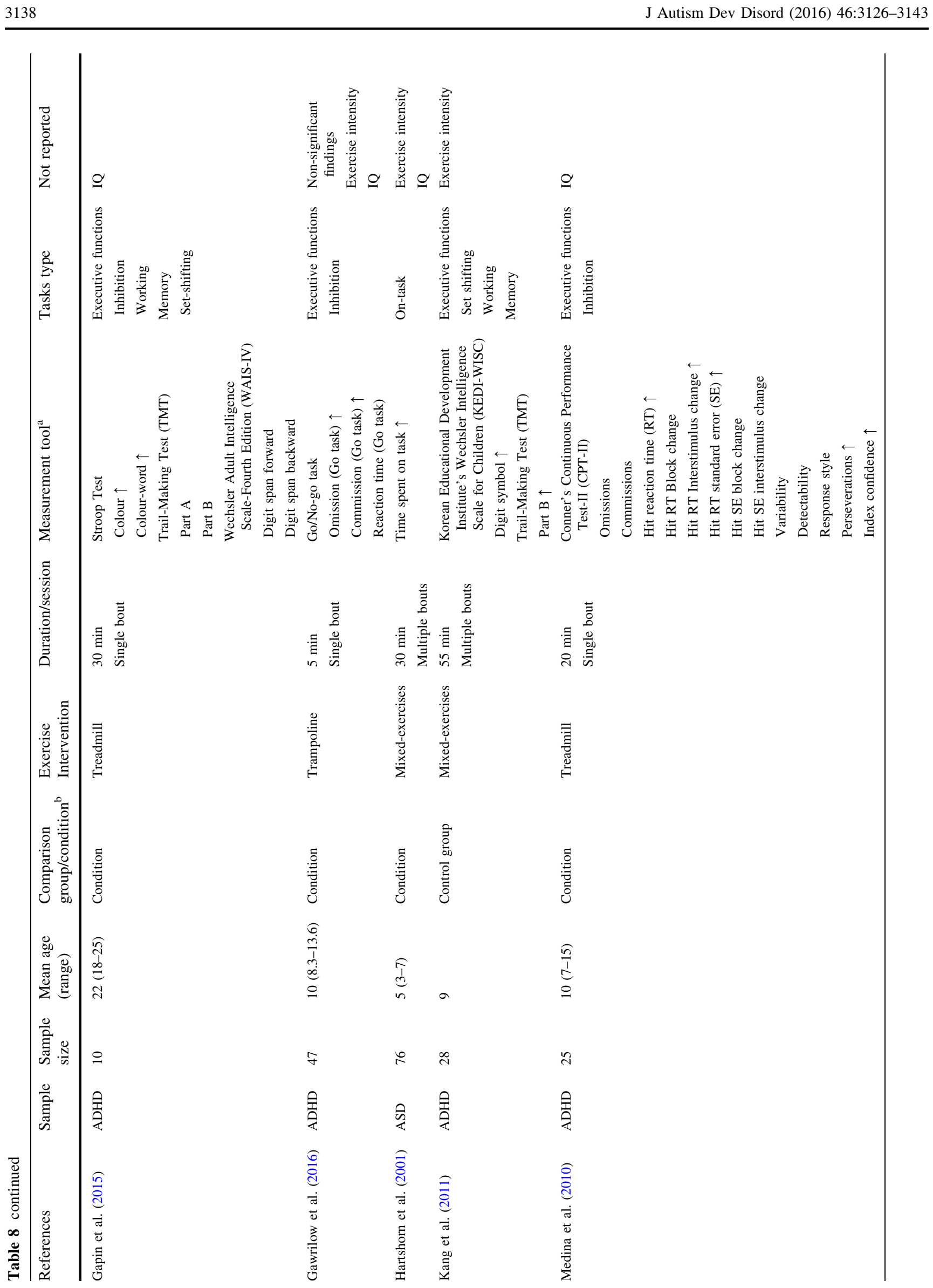

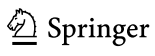




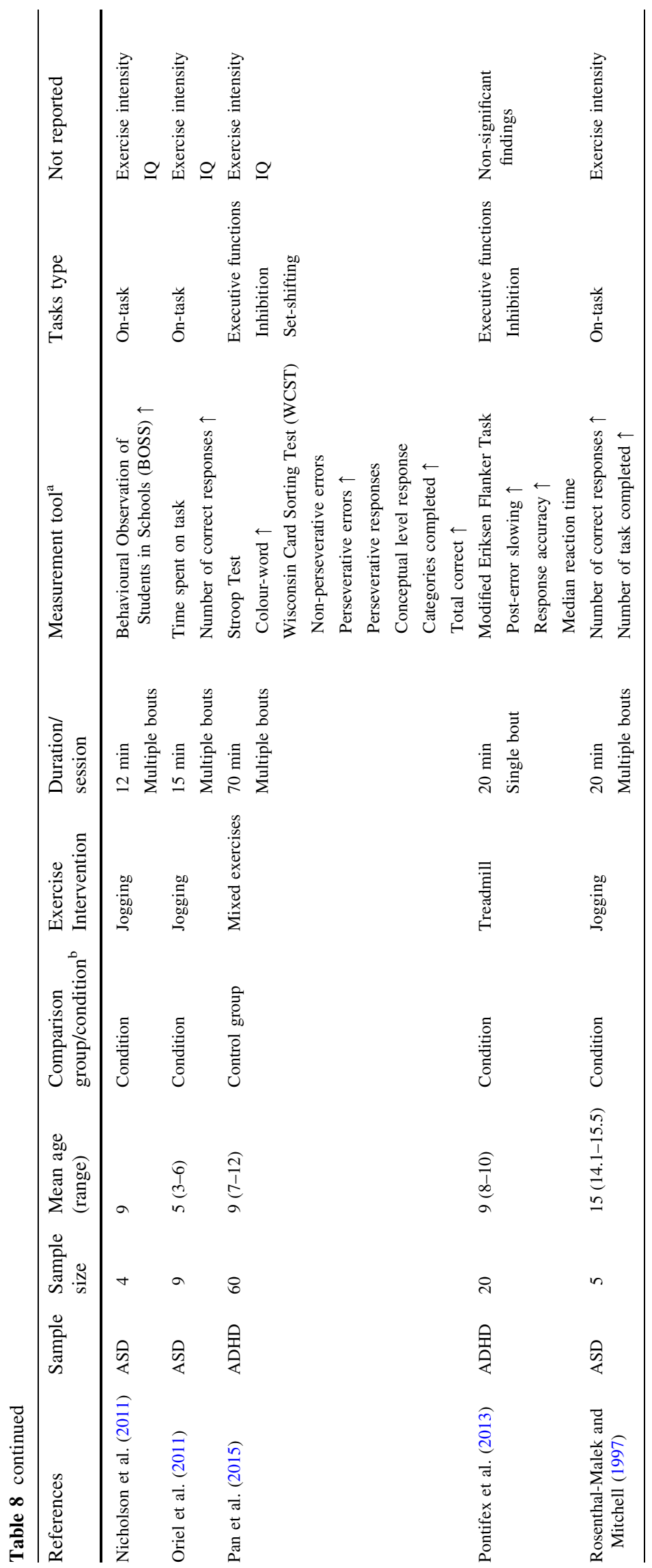




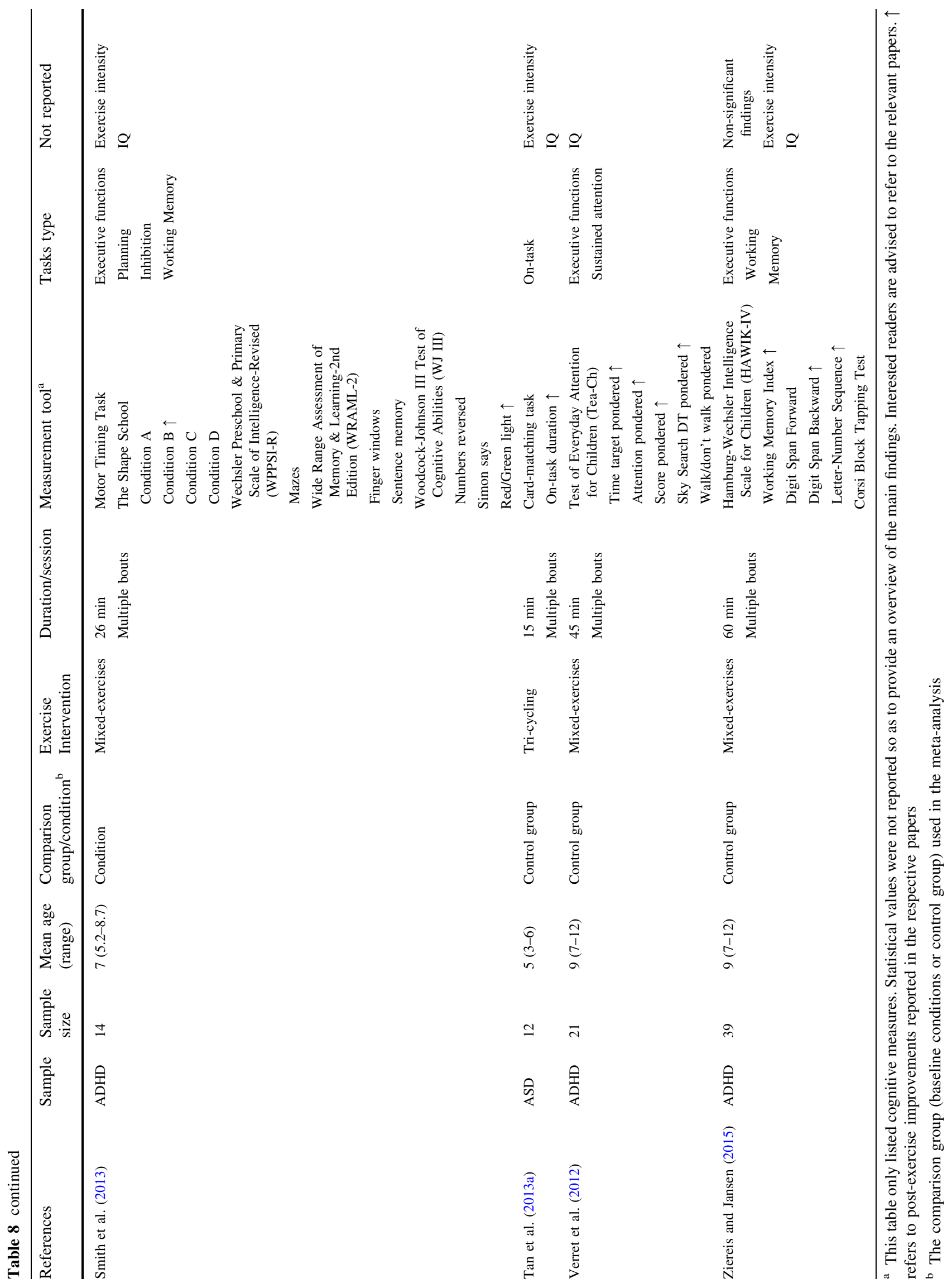




\section{References}

American Psychiatric Association. (2013). Diagnostic and statistical manual of mental disorders. Washington, DC: American Psychiatric Association.

Andersen, P. N., Skogli, E. W., Hovik, K. T., Egeland, J., \& Øie, M. (2015). Associations among symptoms of autism, symptoms of depression and executive functions in children with highfunctioning autism: A 2 year follow-up study. Journal of Autism and Developmental Disorders, 45(8), 2497-2507. doi:10.1007/ s10803-015-2415-8.

Anderson-Hanley, C., Tureck, K., \& Schneiderman, R. L. (2011). Autism and exergaming: effects on repetitive behaviors and cognition. Psychology Research and Behavior Management, 4, 129-137. doi:10.2147/PRBM.S24016.

Audiffren, M. (2009). Acute exercise and psychological functions: A cognitive-energetic approach. In T. McMorris, P. Tomporowski, \& M. Audiffren (Eds.), Exercise and cognitive function (p. 4). Chichester: Wiley.

Becker, L. M. (1997). The effects of exercise versus methylphenidate on attention and behavior in children with attention deficit hyperactivity disorder, predominantly inattentive type (Unpublished doctoral dissertation). University of Alabama at Birmingham, Birmingham, AL

Begg, C. B., \& Mazumdar, M. (1994). Operating characteristics of a rank correlation test for publication bias. Biometrics, 50(4), 1088-1101. doi:10.2307/2533446.

Birchfield, N. (2014). The effects of assisted cycle therapy on executive and motor functioning in young adult females with attention-deficit hyperactivity disorder (Doctoral dissertation, Arizona State University). Retrieved from http://hdl.handle.net/ 2286/R.A. 137400

Borenstein, M., Hedges, L. V., Higgins, J. P. T., \& Rothstein, H. R. (2009). Converting among effect sizes. Introduction to metaanalysis (pp. 45-49). Chichester: John Wiley \& Sons Ltd.

Cassidy, A. R. (2016). Executive function and psychosocial adjustment in healthy children and adolescents: A latent variable modelling investigation. Child Neuropsychology, 22(3), 292-317. doi:10.1080/09297049.2014.994484.

Cerrillo-Urbina, A., García-Hermoso, A., Sánchez-López, M., PardoGuijarro, M., Santos Gómez, J., \& Martínez-Vizcaíno, V. (2015). The effects of physical exercise in children with attention deficit hyperactivity disorder: A systematic review and meta-analysis of randomized control trials. Child: Care. Health and Development, 41, 779-788. doi:10.1111/cch.12255.

Chang, Y.-K., Hung, C.-L., Huang, C.-J., Hatfield, B. D., \& Hung, T.-M. (2014). Effects of an aquatic exercise program on inhibitory control in children with ADHD: A preliminary study. Archives of Clinical Neuropsychology, 29(3), 217-223. doi:10.1093/arclin/acu003.

Chang, Y.-K., Labban, J., Gapin, J., \& Etnier, J. (2012a). The effects of acute exercise on cognitive performance: A meta-analysis. Brain Research, 1453, 87-101. doi:10.1016/j.brainres.2012.02. 068.

Chang, Y.-K., Liu, S., Yu, H.-H., \& Lee, Y.-H. (2012b). Effect of acute exercise on executive function in children with attention deficit hyperactivity disorder. Archives of Clinical Neuropsychology, 27(2), 225-237. doi:10.1093/arclin/acr094.

Chantiluke, K., Christakou, A., Murphy, C. M., Giampietro, V., Daly, E. M., Ecker, C., et al. (2014). Disorder-specific functional abnormalities during temporal discounting in youth with attention deficit hyperactivity disorder (ADHD), autism and comorbid ADHD and autism. Psychiatry Research: Neuroimaging, 223(2), 113-120. doi:10.1016/j.pscychresns.2014.04.006.

Chen, S. F., Chien, Y. L., Wu, C. T., Shang, C. Y., Wu, Y. Y., \& Gau, S. S. (2016). Deficits in executive functions among youths with autism spectrum disorders: An age-stratified analysis. Psychological Medicine, 46(8), 1625-1638. doi:10.1017/S003329 1715002238.

Choi, J. W., Han, D. H., Kang, K. D., Jung, H. Y., \& Renshaw, P. F. (2015). Aerobic exercise and attention deficit hyperactivity disorder: Brain research. Medicine and Science in Sports and Exercise, 47(1), 33-39. doi:10.1249/MSS.0000000000000373.

Chuang, L.-Y., Tsai, Y.-J., Chang, Y.-K., Huang, C.-J., \& Hung, T.M. (2015). Effects of acute aerobic exercise on response preparation in a go/no go task in children with ADHD: An ERP study. Journal of Sport and Health Science, 4(1), 82-88. doi:10.1016/j.jshs.2014.11.002.

Corbett, B. A., Constantine, L. J., Hendren, R., Rocke, D., \& Ozonoff, S. (2009). Examining executive functioning in children with autism spectrum disorder, attention deficit hyperactivity disorder and typical development. Psychiatry Research, 166(2), 210-222. doi:10.1016/j.psychres.2008.02.005.

Craft, D. H. (1983). Effect of prior exercise on cognitive performance tasks by hyperactive and normal young boys. Perceptual and Motor Skills, 56(3), 979-982. doi:10.2466/pms.1983.56.3.979.

Davis, C. L., \& Lambourne, K. (2009). Exercise and cognition in children. In T. McMorris, P. Tomporowski, \& M. Audiffren (Eds.), Exercise and cognitive function (pp. 249-267). Chichester: Wiley.

Eggermont, L. H. P., Milberg, W. P., Lipsitz, L. A., Scherder, E. J. A., \& Leveille, S. G. (2009). Physical activity and executive function in aging: The MOBILIZE Boston study. Journal of the American Geriatrics Society, 57(10), 1750-1756. doi:10. 1111/j.1532-5415.2009.02441.x

Ellis, P. D. (2010). The essential guide to effect sizes: Statistical power, meta-analysis, and the interpretation of research results. New York, NY: Cambridge University Press.

Fedewa, A. L., \& Ahn, S. (2011). The effects of physical activity and physical fitness on children's achievement and cognitive outcomes: A meta-analysis. Research Quarterly for Exercise and Sport, 82(3), 521-535. doi:10.1080/02701367.2011.10599785.

Field, A. (2005). A bluffer's guide to meta-analysis. Psychology Postgraduate Affairs Group Quarterly, (60), 20-39. Retrieved from http://www.statisticshell.com/docs/psypagma.pdf

Field, A. P., \& Gillett, R. (2010). How to do a meta-analysis. British Journal of Mathematical and Statistical Psychology, 63(3), 665-694. doi:10.1348/000711010X502733.

Fisher, R. A. (1921). On the probable error of a coefficient of correlation deduced from a small sample. Metron, 1, 3-32.

Gapin, J. I., Labban, J. D., Bohall, S. C., Wooten, J. S., \& Chang, Y.K. (2015). Acute exercise is associated with specific executive functions in college students with ADHD: A preliminary study. Journal of Sport and Health Science, 4(1), 89-96. doi:10.1016/j. jshs.2014.11.003.

Gargaro, B. A., Rinehart, N. J., Bradshaw, J. L., Tonge, B. J., \& Sheppard, D. M. (2011). Autism and ADHD: How far have we come in the comorbidity debate? Neuroscience and Biobehavioral Reviews, 35(5), 1081-1088. doi:10.1016/j.neubiorev.2010.11.002.

Gawrilow, C., Stadler, G., Langguth, N., Naumann, A., \& Boeck, A. (2016). Physical activity, affect, and cognition in children with symptoms of ADHD. Journal of Attention Disorders, 20(2), 151-162. doi:10.1177/1087054713493318.

Geurts, H. M., Verté, S., Oosterlaan, J., Roeyers, H., \& Sergeant, J. A. (2004). How specific are executive functioning deficits in attention deficit hyperactivity disorder and autism? Journal of Child Psychology and Psychiatry, 45(4), 836-854. doi:10.1111/j. 1469-7610.2004.00276.x.

Goldberg, M. C., Mostofsky, H. S., Cutting, E. L., Mahone, M. E., Astor, C. B., Denckla, B. M., \& Landa, J. R. (2005). Subtle executive impairment in children with autism and children with 
ADHD. Journal of Autism and Developmental Disorders, 35(3), 279-293. doi:10.1007/s10803-005-3291-4.

Hartshorn, K., Olds, L., Field, T., Delage, J., Cullen, C., \& Escalona, A. (2001). Creative movement therapy benefits children with autism. Early Child Development and Care, 166(1), 1-5. doi:10. 1080/0300443011660101.

Hedges, L. V., \& Vevea, J. L. (1998). Fixed-and random-effects models in meta-analysis. Psychological Methods, 3(4), 486-504.

Hill, E. L. (2004). Executive dysfunction in autism. Trends in Cognitive Sciences, 8(1), 26-32. doi:10.1016/j.tics.2003.11.003.

Joshi, G., Faraone, S. V., Wozniak, J., Tarko, L., Fried, R., Galdo, M., \& Biederman, J. (2014). Symptom profile of ADHD in youth with high-functioning autism spectrum disorder: A comparative study in psychiatrically referred populations. Journal of Attention Disorders. doi:10.1177/1087054714543368.

Joshi, G., Petty, C., Wozniak, J., Henin, A., Fried, R., Galdo, M., \& Biederman, J. (2010). The heavy burden of psychiatric comorbidity in youth with autism spectrum disorders: A large comparative study of a psychiatrically referred population. Journal of Autism and Developmental Disorders, 40(11), 1361-1370. doi:10.1007/s10803-010-0996-9.

Kang, K. D., Choi, J. W., Kang, S. G., \& Han, D. H. (2011). Sports therapy for attention, cognitions and sociality. International Journal of Sports Medicine, 32(12), 953-959. doi:10.1055/s0031-1283175.

Kramer, A. F., \& Erickson, K. I. (2007). Capitalizing on cortical plasticity: Influence of physical activity on cognition and brain function. Trends in Cognitive Sciences, 11(8), 342-348. doi:10. 1016/j.tics.2007.06.009.

Lambourne, K., \& Tomporowski, P. (2010). The effect of exerciseinduced arousal on cognitive task performance: A meta-regression analysis. Brain Research, 1341, 12-24. doi:10.1016/j. brainres.2010.03.091.

Leavy, J. E., Bull, F. C., Rosenberg, M., \& Bauman, A. (2011). Physical activity mass media campaigns and their evaluation: A systematic review of the literature 2003-2010. Health Education Research, 26(6), 1060-1085. doi:10.1093/her/cyr069.

Marcus, B., Owen, N., Forsyth, L., Cavill, N., \& Fridinger, F. (1998). Physical activity interventions using mass media, print media, and information technology. American Journal of Preventive Medicine, 15(4), 362-378. doi:10.1016/S0749-3797(98)00079-8.

McMorris, T., \& Hale, B. J. (2012). Differential effects of differing intensities of acute exercise on speed and accuracy of cognition: a meta-analytical investigation. Brain and Cognition, 80(3), 338-351. doi:10.1016/j.bandc.2012.09.001.

McMorris, T., Tomporowski, P., \& Audiffren, M. (2009). Exercise and cognitive function. Chichester: John Wiley \& Sons Ltd.

Medina, J. A., Netto, T. L., Muszkat, M., Medina, A. C., Botter, D., Orbetelli, R., et al. (2010). Exercise impact on sustained attention of ADHD children, methylphenidate effects. ADHD Attention Deficit and Hyperactivity Disorders, 2(1), 49-58. doi:10.1007/s12402-009-0018-y.

Mukaddes, N. M., Hergüner, S., \& Tanidir, C. (2010). Psychiatric disorders in individuals with high-functioning autism and Asperger's disorder: Similarities and differences. The World Journal of Biological Psychiatry, 11(8), 964-971. doi:10.3109/ 15622975.2010 .507785

Netz, Y., Wu, M.-J., Becker, B. J., \& Tenenbaum, G. (2005). Physical activity and psychological well-being in advanced age: A metaanalysis of intervention studies. Psychology and Aging, 20(2), 272-284. doi:10.1037/0882-7974.20.2.272.

Nicholson, H., Kehle, T. J., Bray, M. A., \& Heest, J. V. (2011). The effects of antecedent physical activity on the academic engagement of children with autism spectrum disorder. Psychology in the Schools, 48(2), 198-213. doi:10.1002/pits.20537.
Oriel, K. N., George, C. L., Peckus, R., \& Semon, A. (2011). The effects of aerobic exercise on academic engagement in young children with autism spectrum disorder. Pediatric Physical Therapy, 23(2), 187-193. doi:10.1097/PEP.0b013e318218f149.

Pan, C.-Y., Tsai, C.-L., Chu, C.-H., Sung, M.-C., Huang, C.-Y., \& Ma, W.-Y. (2015). Effects of physical exercise intervention on motor skills and executive functions in children with ADHD: A pilot study. Journal of Attention Disorders. doi:10.1177/ 1087054715569282.

Pennington, B. F., \& Ozonoff, S. (1996). Executive functions and developmental psychopathology. Journal of Child Psychology and Psychiatry, 37(1), 51-87. doi:10.1111/j.1469-7610.1996. tb01380.x.

Petrus, C., Adamson, S. R., Block, L., Einarson, S. J., Sharifnejad, M., \& Harris, S. R. (2008). Effects of exercise interventions on stereotypic behaviours in children with autism spectrum disorder. Physiotherapy Canada, 60(2), 134-145. doi:10.3138/physio. 60.2.134.

Pontifex, M. B., Saliba, B. J., Raine, L. B., Picchietti, D. L., \& Hillman, C. H. (2013). Exercise improves behavioral, neurocognitive, and scholastic performance in children with attentiondeficit/hyperactivity disorder. The Journal of Pediatrics, 162(3), 543-551. doi:10.1016/j.jpeds.2012.08.036.

Randolph, J. J., \& Edmondson, R. S. (2005). Using the binomial effect size display (BESD) to present the magnitude of effect sizes to the evaluation audience. Practical Assessment Research \& Evaluation, 10(14), 1-7. Retrieved from http://pareonline.net/ getvn.asp? $\mathrm{v}=10 \& \mathrm{n}=14 \&$ keyword=r-rosenthal

Ratey, J., \& Loehr, J. (2011). The positive impact of physical activity on cognition during adulthood: A review of underlying mechanisms, evidence and recommendations. Reviews in the Neurosciences, 22(2), 171-185. doi:10.1515/rns.2011.017.

Rosenthal, R. (1979). The file drawer problem and tolerance for null results. Psychological Bulletin, 86(3), 638.

Rosenthal, R. (1991). Meta-analytic procedures for social research. Thousand Oaks, CA: SAGE Publications Inc.

Rosenthal, R., \& Rubin, D. B. (1982). A simple, general purpose display of magnitude of experimental effect. Journal of Educational Psychology, 74(2), 166-169.

Rosenthal-Malek, A., \& Mitchell, S. (1997). Brief report: The effects of exercise on the self-stimulatory behaviors and positive responding of adolescents with autism. Journal of Autism and Developmental Disorders, 27(2), 193-202.

Schreiber, J. E., Possin, K. L., Girard, J. M., \& Rey-Casserly, C. (2014). Executive function in children with attention deficit/ hyperactivity disorder: The NIH EXAMINER battery. Journal of the International Neuropsychological Society, 20(1), 41-51. doi:10.1017/S1355617713001100.

Sibley, B. A., \& Etnier, J. L. (2003). The relationship between physical activity and cognition in children: A meta-analysis. Pediatric Exercise Science, 15(3), 243-256.

Smith, A. L., Hoza, B., Linnea, K., McQuade, J. D., Tomb, M., Vaughn, A. J., et al. (2013). Pilot physical activity intervention reduces severity of ADHD symptoms in young children. Journal of Attention Disorders, 17(1), 70-82. doi:10.1177/108705471 1417395

Sowa, M., \& Meulenbroek, R. (2012). Effects of physical exercise on autism spectrum disorders: A meta-analysis. Research in Autism Spectrum Disorders, 6(1), 46-57. doi:10.1016/j.rasd.2011.09.001.

Speelman, C. P., \& McGann, M. (2013). How mean is the mean? Frontiers in Psychology, 4, 1-12. doi:10.3389/fpsyg.2013. 00451.

Suchy, Y. (2009). Executive functioning: Overview, assessment, and research issues for non-neuropsychologists. Annals of Behavioral Medicine, 37(2), 106-116. doi:10.1007/s12160-009-9097-4. 
Tan, B. W. Z., Cohen, L., \& Pooley, J. A. (2013a). Physical activity: Its implication on attention span and quality of life in children with autism spectrum disorders. GSTF Journal of Law and Social Sciences, 2(2), 108-116. doi:10.5176/2251-2853_2.2.121.

Tan, B. W. Z., Cohen, L., \& Pooley, J. A. (2013b). The relationship between physical activity and cognition and its application to children with autism spectrum disorder. In C. Speelman (Ed.), Enhancing human performance (pp. 22-43). Newcastle: Cambridge Scholars Publishing.

Tomporowski, P. D., Davis, C. L., Miller, P. H., \& Naglieri, J. A. (2008). Exercise and children's intelligence, cognition, and academic achievement. Educational Psychology Review, 20(2), 111-131. doi:10.1007/s10648-007-9057-0.

Tomporowski, P. D., Lambourne, K., \& Okumura, M. S. (2011). Physical activity interventions and children's mental function: An introduction and overview. Preventive Medicine, 52(Supplement), S3-S9. doi:10.1016/j.ypmed.2011.01.028.

Verburgh, L., Königs, M., Scherder, E. J., \& Oosterlaan, J. (2013). Physical exercise and executive functions in preadolescent children, adolescents and young adults: A meta-analysis. British Journal of Sports Medicine, 48(12), 973-979. doi:10.1136/ bjsports-2012-091441.

Verret, C., Guay, M.-C., Berthiaume, C., Gardiner, P., \& Béliveau, L. (2012). A physical activity program improves behavior and cognitive functions in children with ADHD: An exploratory study. Journal of Attention Disorders, 16(1), 71-80. doi:10. 1177/1087054710379735.

Vevea, J. L., \& Woods, C. M. (2005). Publication bias in research synthesis: sensitivity analysis using a priori weight functions. Psychological Methods, 10(4), 428-443. doi:10.1037/1082989X.10.4.428.

Wigal, S. B., Emmerson, N., Gehricke, J.-G., \& Galassetti, P. (2013). Exercise: Applications to childhood ADHD. Journal of Attention Disorders, 17(4), 279-290. doi:10.1177/1087054712454192.

Willcutt, E. G., Doyle, A. E., Nigg, J. T., Faraone, S. V., \& Pennington, B. F. (2005). Validity of the executive function theory of attention-deficit/hyperactivity disorder: A meta-analytic review. Biological Psychiatry, 57(11), 1336-1346. doi:10. 1016/j.biopsych.2005.02.006.

Wolf, F. M. (1986). Meta-analysis: Quantitative methods for research synthesis (Vol. 59). Newbury Park, CA: Sage.

World Health Organisation. (2010). Global recommendations on physical activity for health. Retrieved from http://www.who.int/ en/.

Ziereis, S., \& Jansen, P. (2015). Effects of physical activity on executive function and motor performance in children with ADHD. Research in Developmental Disabilities, 38, 181-191. doi:10.1016/j.ridd.2014.12.005. 